\title{
A supposedly healthy athlete at high-risk of cardioembolic stroke
}

\author{
Massimo Bolognesi* \\ Department of Internal General Medicine, Sports Cardiology Medicine Center, Azienda Unità Sanitaria Locale della Romagna, Italy
}

\begin{abstract}
Cardioembolic stroke is largely preventable, warranting efforts at primary prevention for major risk cardioembolic sources. The goal of sports pre-participation screening is to prevent sudden death in athletes. Here the authors describe the history of an otherwise healthy 60-year-old master athlete who underwent sports pre-participation screening. A resting electrocardiogram showed a pattern suggestive of recent anterior myocardial infarction. A focused echocardiogram showed left ventricular apical akinesis with a small and mobile apical thrombus protruding into the left ventricular cavity. The risk of cardioembolic stroke was very high. This report highlights the importance of pre-participation screening to prevent sudden cardiac death.
\end{abstract}

\section{Introduction}

Cardioembolic stroke is largely preventable, warranting efforts at primary prevention for major risk cardioembolic sources [1]. Echocardiography has allowed clinicians to better characterize well-established sources of embolism and to discover other potential etiologies of cardioembolic stroke [2]. Common high-risk cardioembolic conditions are atrial fibrillation, recent myocardial infarction, mechanical prosthetic valve, dilated cardiomyopathy, and valvular heart disease. Emboli arising from the cardiac chambers are often of considerable size, and therefore more likely to cause severe stroke, disability and death [3]. Two-dimensional trans-thoracic echocardiography (TTE) is the usual initial cardiac imaging modality. TTE reliably detects left ventricular wall motion abnormalities, and left ventricular thrombus. Nowadays, its use in sports cardiology medicine is mandatory to screen for potentially lethal underlying heart conditions including certain structural abnormalities and undetected myocardial infarction that might be complicated by left ventricular thrombus even in an asymptomatic athletes [4]. The most likely cause of sudden cardiac death in master athletes beyond the young adult age is asymptomatic or silent coronary artery disease [5]. Case reports in the literature about this entity are limited [6]. This report illustrates an unusual high-risk situation that would have gone undetected in this asymptomatic athlete had sports pre-participation screening not been done.

\section{Case report}

The patient is an otherwise healthy 60 -year-old amateur bicycle rider who came for sports pre-participation screening to the Sports Cardiology Medicine Center in Cesena, Italy. His family history was unremarkable. Physical examination was completely normal. His previous sports screening physical examination was normal. Peaked T waves were seen in lateral chest leads at that time, but there was no evidence of anterior infarction (Figure 1). As the patient was absolutely asymptomatic, this initial ECG was felt to represent a repolarization variant. However, on return two years later, his ECG now clearly indicates that anterior myocardial infarction has occurred in the interim (Figure 2). Echocardiogram using classical and magnified apical 4 chamber view showed overall good ventricular function, as well as left ventricular apical akinesis with a small mobile apical thrombus (Figure 3) protruding into the left ventricular cavity. Anticoagulation therapy was started, as the risk of cardioembolic stroke was felt to be very high. The patient underwent myocardial revascularization, and within 10 days of anticoagulation the clot had dissolved.

\section{Discussion}

Asymptomatic myocardial infarction is a rare event in athletes.

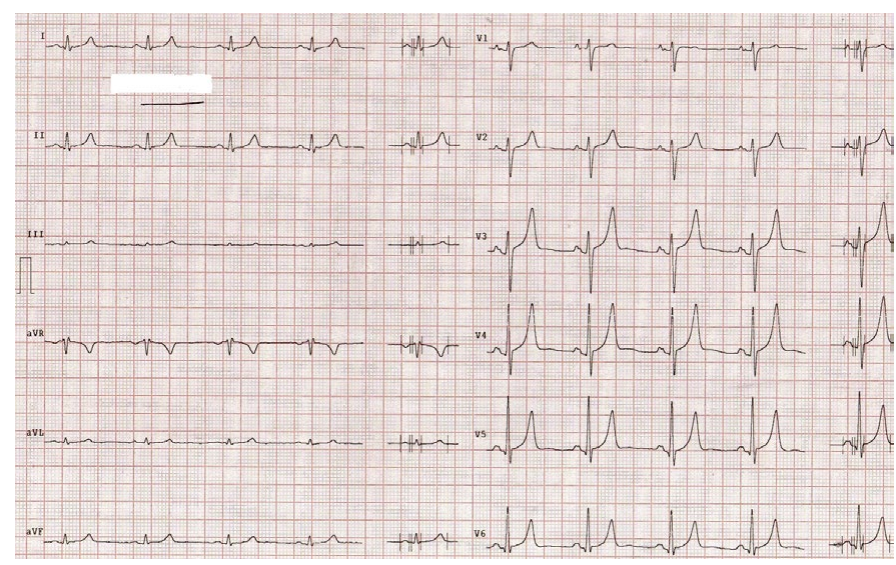

Figure 1. The patient's original resting ECG done in 2013.

Correspondence to: Massimo Bolognesi, MD, Department of Internal General Medicine, Sports Cardiology Medicine Center, Azienda Unità Sanitaria Locale della Romagna Via Ungaretti, 49447521 Cesena, Italy, Tel: +390547645074; E-mail: massbolo1@tin.it

Key words: cardioembolic stroke, athlete, echocardiography, apical akinesis, thrombus

Received: April 27, 2016; Accepted: May 14, 2016; Published: May 19, 2016 


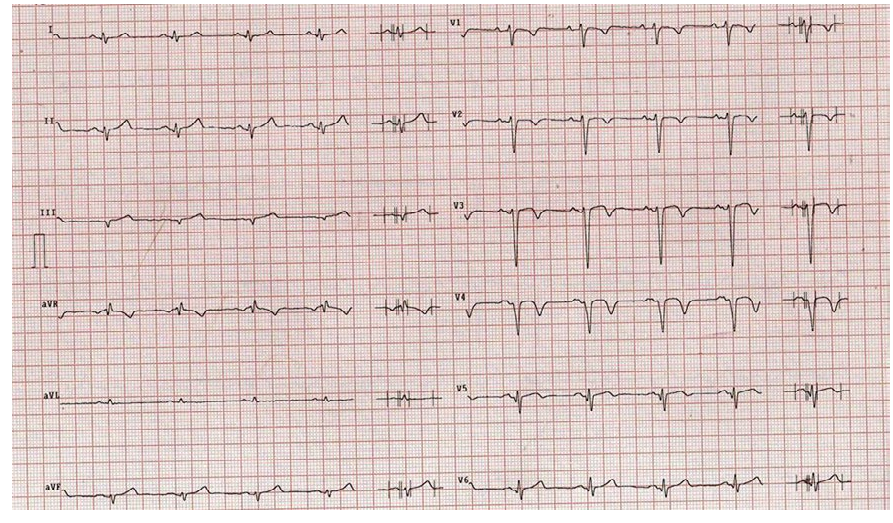

Figure 2. The patient's follow-up ECG done in 2015. Anterior myocardial infarction has occurred in the interim.
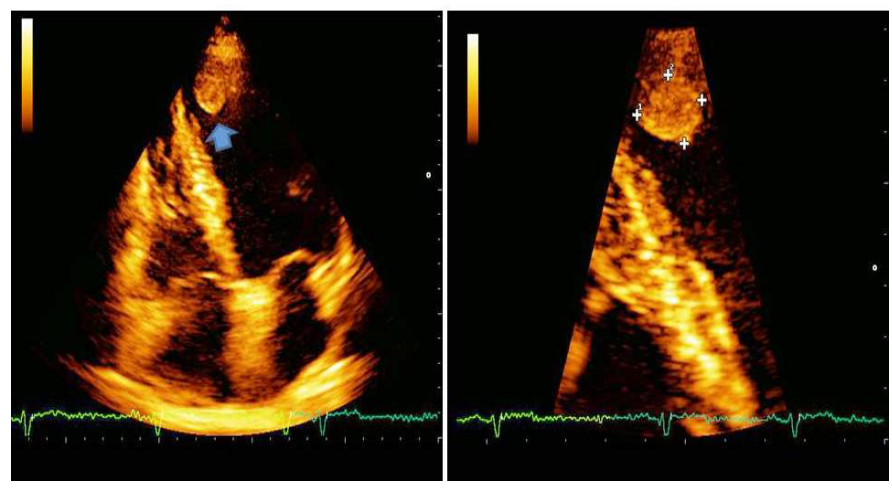

Figure 3. Echocardiographic findings of left ventricular apical thrombus (see arrow) by classical and magnified apical 4 chamber view.
This case illustrates that it nevertheless can happen, and may present with the extremely high-risk lesion of an apical thrombus. Learning points from this case include the following: 1) silent or asymptomatic ischemic heart disease may occur even in master athletes; 2) a noncomplicated myocardial infarction may be compatible with surprisingly good systolic function after the event when the patient is a well trained athlete; and 3) obtaining an echocardiogram is especially important following anterior myocardial infarction to rule out apical thrombus.

\section{Conclusion}

This report highlights the importance of pre-participation screening to prevent sudden cardiac death by presenting a case in which asymptomatic infarction with resultant mobile apical thrombus was only picked up by such screening.

\section{References}

1. Hart RG, Albers G, Koudstaal P (1998) Cardioembolic stroke. Ginsberg M, Bogousslavsly J(Eds.), Cerebrovascular Disease: Pathophysiology, Diagnosis and Management. Blackwell Science; 1392-429.

2. Wessler BS, Kent DM (2015) Controversies in cardioembolic stroke. Curr Treat Options Cardiovasc Med 17: 358. [Crossref]

3. Arboix A, Alió J (2010) Cardioembolic stroke: clinical features, specific cardiac disorders and prognosis. Curr Cardiol Rev 6: 150-161. [Crossref]

4. Delewi R, Zijlstra F, Piek JJ (2012) Left ventricular thrombus formation after acute myocardial infarction. Heart 98: 1743-1749. [Crossref]

5. TunstallPedoe DS (2007) Marathon cardiac deaths: the london experience. Sports Med 37: 448-450. [Crossref]

6. Bolognesi M, Bolognesi D (2014) Asymptomatic Ischemic Heart Disease in a 45-yearold Male Athlete: A Case Report. J Gen Pract 2: 139.

Copyright: (C2016 Bolognesi M. This is an open-access article distributed under the terms of the Creative Commons Attribution License, which permits unrestricted use, distribution, and reproduction in any medium, provided the original author and source are credited. 